\title{
Strength and physiological response to exercise in patients with chronic fatigue syndrome
}

\author{
Kathy Y Fulcher, Peter D White
}

\begin{abstract}
Objective-To measure strength, aerobic exercise capacity and efficiency, and functional incapacity in patients with chronic fatigue syndrome (CFS) who do not have a current psychiatric disorder.

Methods-Sixty six patients with CFS without a current psychiatric disorder, 30 healthy but sedentary controls, and 15 patients with a current major depressive disorder were recruited into the study. Exercise capacity and efficiency were assessed by monitoring peak and submaximal oxygen uptake, heart rate, blood lactate, duration of exercise, and perceived exertion during a treadmill walking test. Strength was measured using twitch interpolated voluntary isometric quadriceps contractions. Symptomatic measures included physical and mental fatigue, mood, sleep, somatic amplification, and functional incapacity.
\end{abstract}

Results-Compared with sedentary controls, patients with CFS were physically weaker, had a significantly reduced exercise capacity, and perceived greater effort during exercise, but were equally unfit. Compared with depressed controls, patients with CFS had significantly higher submaximal oxygen uptakes during exercise, were weaker, and perceived greater physical fatigue and incapacity. Multiple regression models suggested that exercise incapacity in CFS was related to quadriceps muscle weakness, increased cardiovascular response to exercise, and body mass index. The best model of the increased exercise capacity found after graded exercise therapy consisted of a reduction in submaximal heart rate response to exercise.

Conclusions-Patients with CFS were weaker than sedentary and depressed controls and as unfit as sedentary controls. Low exercise capacity in patients with CFS was related to quadriceps muscle weakness, low physical fitness, and a high body mass ratio. Improved physical fitness after treatment was associated with increased exercise capacity. These data imply that physical deconditioning helps to maintain physical disability in CFS and that a treatment designed to reverse deconditioning helps to improve physical function.

(F Neurol Neurosurg Psychiatry 2000;69:302-307)

Keywords: chronic fatigue syndrome; exercise; incapacity; muscle strength; fitness
Chronic fatigue syndrome (CFS) is characterised by postexertional or persistent fatigue, with consequent disability. ${ }^{2}$ Until recently no abnormalities of muscle physiology or metabolism that could explain the fatigue had been reported. ${ }^{3}$ Lane et al found an increased lactic acid response to exercise in $37 \%$ of patients with CFS and these patients were particularly likely to have type II muscle fibre predominance. ${ }^{4}$ Barnes et al also showed increased metabolic acidification with exercise in a smaller minority, but suggested that "detraining" due to inactivity was the likely cause. ${ }^{5}$ Physical deconditioning may contribute to the fatigue of CFS, ${ }^{6}$ possibly as a result of a less active lifestyle. ${ }^{7-9}$ An empirically derived model of fatigue and disability in CFS suggested that the amount of physical activity had an important effect on fatigue and an even stronger effect on disability. ${ }^{10}$ Both lower exercise capacity and lower peak oxygen consumption have been reported in patients with $\mathrm{CFS}^{611}$ and related conditions ${ }^{7}$ compared with controls. Both higher heart rates ${ }^{67}$ and perceived exertion ${ }^{6}{ }^{11-13}$ have been reported with submaximal exercise when compared with either healthy active or healthy sedentary controls.

All these studies used criteria for CFS that included psychiatric disorders such as major depressive disorder. ${ }^{12}$ There have been no studies of the physiology of CFS in the absence of comorbid psychiatric disorders. The aim of this study was to examine both physiological and symptomatic measures in patients with CFS without psychiatric disorders, and to compare them with both healthy but sedentary subjects and patients with major depressive disorders.

\section{Methods}

Sixty six outpatients fulfilling the Oxford criteria for CFS were recruited through a fatigue clinic at a general hospital department of psychiatry. ${ }^{1}$ All of these patients had agreed to participate in a trial of graded exercise therapy. ${ }^{14}$ Seventy seven other potential patients with CFS, who also had a current psychiatric disorder or significant insomnia, were excluded by the structured clinical interview DSM IIIR (SCID), ${ }^{15}$ because of their separate effects on exercise induced fatigue. ${ }^{16} 17$ Five potential patients refused to participate and five were judged too incapacitated to participate in the study. We included patients with comorbid phobias, because of evidence that phobias do not exacerbate fatigue. ${ }^{18}$ Patients with neurasthenia and unspecified somatoform disorder were included as these diagnoses are often synonymous with CFS. 
Thirty healthy but sedentary controls were recruited through posters and personal referral from staff at St Bartholomew's hospital and its medical and dental school. All volunteers completed an activity questionnaire. This detailed the number of episodes of physical exercise in the previous 3 months and the number of times they took part in strenuous, moderate, or mild activity in the average week. We also assessed their attitude to activity - that is, whether they considered themselves adequately active or sedentary. Only those subjects who took part in no strenuous activity and exercised moderately less than once a week were accepted for entry into the study.

Fifteen patients with major depressive disorder (DSM-IIIR criteria) ${ }^{19}$ were recruited from attendees at the department of psychological medicine.

Ethical approval was obtained from the district research ethics committee and all subjects gave valid and informed consent before entering the study.

SYMPTOMATIC ASSESSMENTS

All questionnaires were completed by subjects before the physiological assessments. Fatigue was measured with self rated visual analogue scales measuring physical, mental, and total fatigue, ${ }^{18}$ and a self rated 14 item fatigue questionnaire. $^{20}$ The hospital anxiety and depression scale was used to assess anxiety and depression. ${ }^{21}$ The Pittsburgh sleep quality index measured quality and quantity of sleep. ${ }^{22}$ The 36 item short form health survey self rated questionnaire measured general health, physical, mental and social capacity. ${ }^{23}$ The five item Barsky self rated somatic amplification scale assessed the tendency to amplify specific body sensations. $^{24}$

\section{PHYSIOLOGICAL ASSESSMENT}

Skinfold measurements were taken at four sites to give a total score in millimetres as an indication of body fat composition. Forced vital capacity (FVC) and forced expired volume in 1 second $\left(\mathrm{FEV}_{1}\right)$ were measured with a Vitalograph spirometer. Normal ranges were taken from Vitalograph Ltd spirometric tables. ${ }^{25}$ Maximum voluntary isometric contraction of the quadriceps muscle of the dominant leg was measured, with percutaneous twitch interpolation to ensure maximal activation and to override central inhibition. ${ }^{26}$ Subjects were seated in a specially adapted rigid, straight backed chair, and asked to push against a strap placed around their ankle. The best of five repetitions was recorded and we noted whether it was with or without stimulation. All subjects were thor-

Table 1 Characteristics of patients and controls

\begin{tabular}{lllll}
\hline Variable & $\begin{array}{l}\text { CFS patients } \\
n=66\end{array}$ & $\begin{array}{l}\text { Depressed patients } \\
n=15\end{array}$ & $\begin{array}{l}\text { Sedentary controls } \\
n=30\end{array}$ & $\begin{array}{l}\text { ANOVA } \\
p \text { Value }\end{array}$ \\
\hline Age (y) & $37.2(10.7)$ & $35.3(10)$ & $36.8(11.1)$ & 0.31 \\
Height (cm) & $169.5(163-176)$ & $169(162-173)$ & $163(159-168)$ & 0.07 \\
Weight (kg) & $67.2(13)$ & $70.1(12.1)$ & $67.9(14.2)$ & 0.27 \\
Body mass index & $22.7(20.0-25.4)$ & $23.7(20.7-26.8)$ & $23.4(20.8-26.0)$ & 0.38 \\
Total skinfold (mm) & $53(34-71)$ & $42(30-54)$ & $51(39-63)$ & 0.23 \\
Female:male ratio (\%) & $49 / 17(74)$ & $10 / 5(66)$ & $22 / 8(73)$ & $0.09^{\star}$ \\
\hline
\end{tabular}

Values are mean (SD)) or median (interquartile range).

${ }^{\star} \chi^{2}$ analysis was used. oughly familiarised with the treadmill before the walking test, carried out at a constant speed of $5 \mathrm{kph}$ and a gradient increase of $2.5 \%$ every 2 minutes. Expired air was collected through a lightweight mouthpiece and analysed for oxygen, carbon dioxide, and minute ventilation. Heart rate was monitored using a three lead ECG. Ratings of perceived exertion (RPEs) were recorded with the Borg 15 point scale in the last 30 seconds of each treadmill stage. ${ }^{27}$ The capillary blood lactate concentration was measured at a Borg RPE rating of 14 (between "somewhat hard" and "hard") and 3 minutes after completion of the test. The test was terminated at volitional fatigue whereby subjects were encouraged to continue to their maximum. Peak levels for all variables were recorded at this point. Age predicted maximum heart rates were calculated from the formula 210- (age $\times 0.65) .{ }^{28}$ Because patients with CFS were about to be entered into a therapeutic trial, it was not possible to assess them blindly, but care was taken to ensure that the same explanation and encouragement were given to all subjects.

\section{ANALYSIS}

Analysis of variance (ANOVA) or a KruskalWallis analysis were used to compare the means or medians between the three groups. When either test indicated a significant difference between the three groups, either Student's $t$ tests or Mann-Whitney $U$ tests were used to compare the means or medians between two groups of interest. We compared the submaximal responses to exercise between groups by comparing the area under the curve of between 6 and 12 minutes inclusively on the treadmill by Kruskal-Wallis one way ANOVA. We examined the possibly confounding effects of taking antidepressant medications in the CFS group. Forward stepwise multiple regression models were calculated on patients with CFS alone, to explore the relation between the dependent variable of exercise capacity and the independent variables of mood, sleep, body mass ratio, strength, and physiological responses to exercise.

\section{Results}

There were no significant differences between the three groups in age, height, weight, sex, or body mass index (table 1). The median (range) duration of illness for the patients with CFS was $2.7(0.6-19.0)$ years which was significantly longer than the depressed patients $(1.2$ $(0.25-12.0)$ years $(p<0.05))$. Thirty two $(48 \%)$ of the patients with CFS were taking medication at the time of assessment; 20 patients were taking normal doses of antidepressants (having previously been treated for a depressive illness), 10 were taking antidepressants at low (hypnotic) doses, and two were on other medication. Thirteen $(87 \%)$ patients in the depressed group were on normal doses of antidepressant medication, which was significantly more than the proportion of patients with CFS taking medication $\left(\chi^{2}=8.4, \mathrm{df}=2, \mathrm{p}<0.05\right)$. 
Table 2 Physiological measurements

\begin{tabular}{|c|c|c|c|c|}
\hline Variable & $\begin{array}{l}\text { CFS patients } \\
n=66\end{array}$ & $\begin{array}{l}\text { Depressed patients } \\
n=15\end{array}$ & $\begin{array}{l}\text { Sedentary controls } \\
n=30\end{array}$ & $\begin{array}{l}\text { ANOVA p } \\
\text { Value }\end{array}$ \\
\hline Test duration (min) & $10(8-12)$ & $12(9-15)$ & $13.5(11-16)$ & 0.001 \\
\hline Peak oxygen uptake $(\mathrm{ml} / \mathrm{kg} / \mathrm{min})$ & $30.6(8.2)$ & $30.9(6.7)$ & $34.1(6.8)$ & 0.13 \\
\hline Maximum ventilation $(1 / \mathrm{min})$ & $69.8(57-83)$ & $70.0(56-84)$ & $82.4(66-87.5)$ & 0.09 \\
\hline Maximum heart rate (beats/min) & $171(18)$ & $172(18)$ & $182(14)$ & 0.01 \\
\hline Recovery heart rate (beats/min) & $117(17))$ & $113(22)$ & $109(17)$ & 0.17 \\
\hline Predicted maximum heart rate $(\%)$ & $91.6(8.8)$ & $91.5(7.6)$ & $97.5(5.2)$ & 0.002 \\
\hline Submaximal blood lactate $(\mathrm{mmol} / \mathrm{l})$ & $2.8(1.6-4.0)$ & $2.8(1.7-3.8)$ & $3.5(2.2-4.8)$ & 0.005 \\
\hline Post-test blood lactate $(\mathrm{mmol} / \mathrm{l})$ & $5.4(2.2)$ & $6.3(2.7)$ & $6.8(2.1)$ & 0.02 \\
\hline Maximum voluntary quadriceps contraction (Newtons) & $310(234-386)$ & $401(315-487)$ & $442(364-518)$ & 0.0001 \\
\hline
\end{tabular}

Values are mean (SD)) or median (interquartile range).

PHYSIOLOGICAL MEASURES

Results of the physiological measures are shown in table 2 . There were significant intergroup differences in test duration, maximum heart rate, percentage of age predicted maximum heart rate reached, peak blood lactate, blood lactate at RPE 14, and maximum quadriceps strength. Differences in peak oxygen uptake $\left(\mathrm{VO}_{2}\right)$ and maximum ventilation were not statistically significant. Resting lung function measures were all within normal ranges with no significant differences between groups (data not shown).

Compared with the healthy sedentary controls, the patients with CFS had a significantly lower aerobic capacity (peak $\left.\mathrm{VO}_{2}\right)(\mathrm{p}=0.05)$, maximum ventilation $(\mathrm{p}=0.05)$, and peak blood lactate $(p<0.01)$. The CFS group terminated the test at a significantly lower maximum heart rate $(p<0.01)$, representing $91.5 \%$ of their age predicted maximum, compared with $97.5 \%$ in the sedentary group. All these results were probably due to the significantly shorter time on the treadmill $(\mathrm{p}<0.001)$. The depressed subjects had a similarly low exercise capacity as the CFS group, but the difference between the depressed and sedentary groups in peak $\mathrm{VO}_{2}$ did not reach significance. Similarly to the patients with CFS, the depressed group reached only $91.6 \%$ of their age predicted maximum heart rate, which was significantly lower than the sedentary group $(p=0.05)$. Independently of test duration, the blood lactate at RPE 14 was significantly lower than in the sedentary group in both patients with CFS $(p<0.005)$ and depressed patients $(\mathrm{p}<0.05)$.

The patients with CFS were significantly weaker than the sedentary subjects $(p<0.001)$, with a trend to being weaker than the depressed patients $(p=0.06)$. Twitch interpolation was required to record the maximum strength in $23 / 66(35 \%)$ of the CFS cases, $6 / 30(20 \%)$ of the sedentary controls, and $2 / 15(13 \%)$ of the depressed controls $\left(\chi^{2}=4.09,2 \mathrm{df}, \mathrm{p}=0.12\right)$.

Oxygen uptake $\left(\mathrm{VO}_{2}\right)$, heart rate, and perceived exertion at all stages of the treadmill test are shown in figs $1-3$, respectively. There were group effects for submaximal perceived exertion $(\mathrm{RPE})\left(\chi^{2}=10.64,2 \mathrm{df}, \mathrm{p}<0.005\right), \mathrm{VO}_{2}$ $\left(\chi^{2}=8.21,2 \mathrm{df}, \mathrm{p}<0.02\right)$, and heart rate $\left(\chi^{2}=5.52,2 \mathrm{df}, \mathrm{p}=0.06\right)$. Both the patients with CFS and the depressed patients had higher submaximal perceived exertion scores (RPE) than the sedentary controls (Mann-Whitney test $U=178.5, \mathrm{p}=0.004$, and $U=49, \mathrm{p}=0.01$, respectively, fig 1 ). Both the patients with CFS

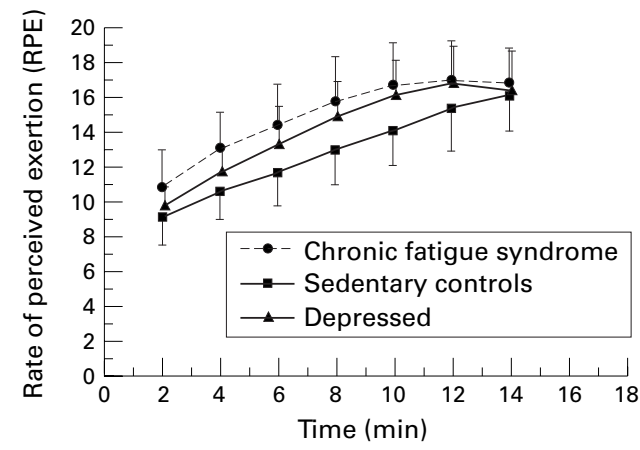

Figure 1 Submaximal perceived exertion rating during the treadmill walking test. Values are mean (SD).

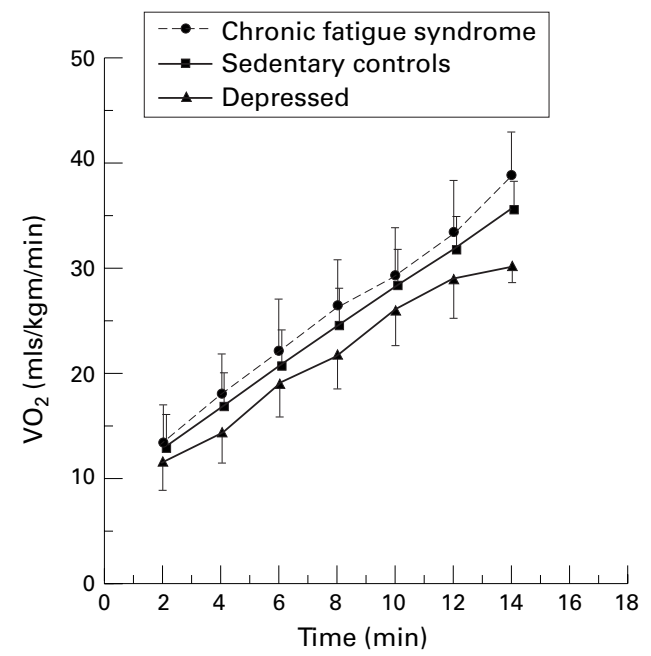

Figure 2 Submaximal oxygen uptake during the treadmill walking test. Values are mean (SD).

and sedentary subjects had higher submaximal $\mathrm{VO}_{2}$ than the depressed patients $(U=56.5$, $\mathrm{p}=0.009$ and $U=49, \mathrm{p}=0.02$, respectively, fig $2)$. The sedentary controls had higher submaximal heart rates than the depressed group ( $U=72, \mathrm{p}=0.05$ ), but the difference between the CFS and depressed groups did not reach significance $(p=0.16$, fig 3$)$.

SYMPTOMATIC MEASURES

Total, physical, and mental fatigue were significantly greater in the depressed patients and patients with CFS, compared with the sedentary controls (table 3). Similarly, the depressed patients had significantly higher HAD depression and anxiety scores than the other two groups. Although there were no significant differences in either total or mental fatigue between the CFS and depressed groups, the patients with CFS were significantly more 


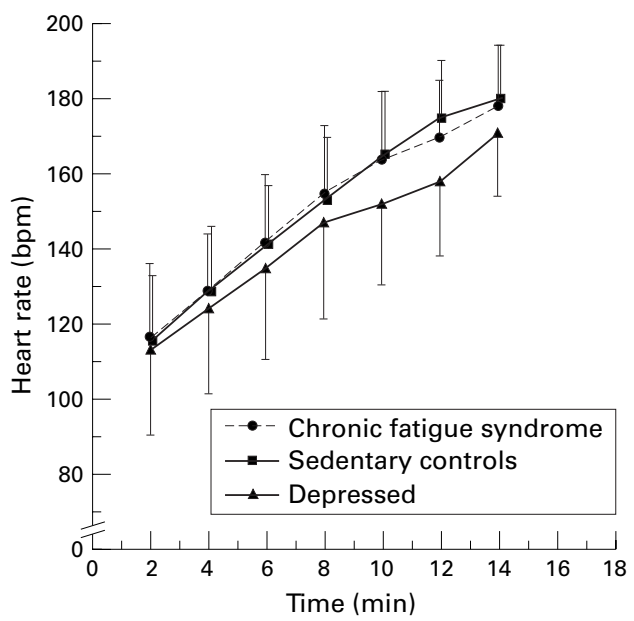

Figure 3 Submaximal exercise heart rate during the treadmill walking test. Values are mean (SD).

physically fatigued than the depressed patients $(p<0.001)$, but this difference was equally composed of both postexertional and persistent physical fatigue. The mean (95\% CI) differences between the CFS and depressed groups were $12(3-21) \mathrm{mm}$ for postexertional physical fatigue and $15(6-23) \mathrm{mm}$ for persistent physical fatigue. The CFS group had a significantly higher somatic amplification score than the sedentary group $(\mathrm{p}<0.008)$, but only a non-significant trend for higher scores than the depressed group $(p=0.11)$. The difference between the CFS and sedentary groups was mainly composed of sensitivity to noise and general awareness of body sensations, with no difference found in sensitivity to pain. The difference between the CFS and depressed groups was mostly composed of the depressed subjects being less aware of pain and hunger.

The two patient groups were significantly more incapacitated than the sedentary controls on all SF-36 measures $(\mathrm{p}<0.001)$, except that the patients with CFS were not significantly different in emotional or mental function. The unexpected results from the SF-36 were the significantly lower scores of physical functioning and role in patients with CFS, when com-

Table 3 Symptomatic measurements

\begin{tabular}{lllll}
\hline Variable & $\begin{array}{l}\text { CFS patients } \\
n=66\end{array}$ & $\begin{array}{l}\text { Depressed } \\
\text { patients } n=15\end{array}$ & $\begin{array}{l}\text { Sedentary } \\
\text { controls } n=30\end{array}$ & $\begin{array}{l}\text { ANOVA p } \\
\text { Value }\end{array}$ \\
\hline HAD anxiety score & $5(2-8)$ & $10(7-13)$ & $7(4-10)$ & 0.0005 \\
HAD depression score & $5(2.5-7.5)$ & $11(9-13)$ & $5.5(1.5-9.5)$ & $<0.0001$ \\
Somatic amplification score & $10(7-13)$ & $7(4.5-9.5)$ & $5.5(2-9)$ & 0.03 \\
PSQI sleep score & $6(4.5-7.5)$ & $9(5-13)$ & $5(2-8)$ & 0.08 \\
Total fatigue & $318(47)$ & $296(67)$ & $213(52)$ & $<0.0001$ \\
Mental fatigue & $155(132-178)$ & $150(120-181)$ & $106(89-123)$ & $<0.0001$ \\
Physical fatigue & $175(159-192)$ & $138(107-169)$ & $101(90-122)$ & $<0.0001$ \\
Chalder fatigue & $30(25.5-34.5)$ & $27(20-34)$ & $14(9.5-18.5)$ & $<0.0001$ \\
SF-36 physical function & $45(35-55)$ & $85(70-100)$ & $93(73-100)$ & $<0.0001$ \\
SF-36 role physical & $0(0-25)$ & $50(0-100)$ & $100(75-100)$ & $<0.0001$ \\
SF-36 bodily pain & $62(41-83)$ & $44(31-52)$ & $84(74-94)$ & $<0.0001$ \\
SF-36 general health & $37(25-50)$ & $45(23-68)$ & $67(55-79)$ & $<0.0001$ \\
SF-36 vitality & $25(15-35)$ & $20(5-35)$ & $60(46-64)$ & $<0.0001$ \\
SF-36 social function & $38(13-63)$ & $50(31-69)$ & $100(84-100)$ & $<0.0001$ \\
SF-36 role emotional & $67(34-100)$ & $0(0-33)$ & $100(83-100)$ & $<0.0001$ \\
SF-36 mental health & $64(53-75)$ & $36(22-50)$ & $74(61-88)$ & $<0.0001$ \\
\end{tabular}

Values are mean (SD) or median (interquartile range).

Normal or usual scores are 14 for Chalder questionnaire, 200 for total fatigue, and 100 for physical and mental fatigue scores (visual analogue scales). 100 is the maximum (full capacity) SF-36 score for subscales. A score $<8$ on hospital anxiety and depression (HAD) scale is considered non-pathological. A Pittsburgh sleep quality index (PSQI) score $<6$ is considered nonpathological. pared with those patients with depression $(\mathrm{p}<0.001$ for both variables), but there were no significant differences between these two groups in vitality, bodily pain, general health, and social functioning. The depressed subjects were significantly more incapacitated in emotional and mental functioning than the patients with CFS $(p<0.001)$. The belief that a virus was the cause of a patient's illness had no significant effect on severity of fatigue or perceived functional incapacity.

CONFOUNDING EFFECTS OF ANTIDEPRESSANTS

Taking antidepressants had no significant effects on illness duration, fatigue, mood, sleep quality, or physical function scores (data not shown). Those taking antidepressants had a higher median (interquartile range) body mass ratio (25.0 (21.4-27.6) versus 22.0 (20.025.2) $(\mathrm{p}=0.03)$ and a shorter median (interquartile range) time on the treadmill (8.6 $(6-11)$ versus $11.0(8-12)$ minutes) $(p=0.02)$. They also had a significantly lower mean (SD) peak $\mathrm{VO}_{2} \quad(27.9 \quad(9.0)$ versus $32.8 \quad(7.0)$ $\mathrm{ml} / \mathrm{kg} / \mathrm{min})(\mathrm{p}=0.02)$. There were no significant differences in maximum heart rate or quadriceps strength (data not shown). Similarly, there were no significant differences in submaximal measures of heart rate, $\mathrm{VO}_{2}$, or RPE (data not shown).

REGRESSION MODELS

We obtained satisfactory models (reasonable variance explained and normally distributed residuals) for exercise tolerance (time spent on the treadmill) both before and after treatment with graded exercise therapy. ${ }^{14}$ We also successfully modelled the increased exercise tolerance which occurred after treatment.

The final model for exercise tolerance (time spent on the treadmill; logged (base 10) to normalise the distribution) was composed of quadriceps strength $(\beta=0.518, \quad t=5.42$, $\mathrm{p}<0.001)$, body mass index $(\beta=-0.405$, $t=-4.32, \mathrm{p}<0.001)$, and heart rate at 6 minutes on the treadmill $(\beta=-0.280, t=-2.95$, $\mathrm{p}=0.004)$. This model accounted for $53 \%$ of the variance in exercise tolerance. We further tested this model twice: at supervised treatment cessation ( 3 months after starting treatment) and after a further 3 months, by entering only these three variables, and the model was confirmed. At treatment cessation, the final model for exercise capacity (explaining $49 \%$ of the variance) was heart rate at 4 minutes on the treadmill $(\beta=-0.478, \quad t=-4.66, \quad \mathrm{p}<0.001)$, quadriceps strength $\quad(\beta=0.308, \quad t=2.97$, $\mathrm{p}=0.004)$, and then body mass index $(\beta=-0.305, t=-2.95, \mathrm{p}=0.005)$. At 3 months follow up after treatment cessation, the final model (with $63 \%$ of the variance explained) was heart rate at 4 minutes on the treadmill $(\beta=-0.428, t=-4.21, \mathrm{p}<0.001)$, quadriceps strength $(\beta=0.436, t=4.44, p<0.001)$, and then body mass index $(\beta=-0.343, t=-3.43$, $\mathrm{p}=0.001)$.

The final model $(30 \%$ of the variance explained) for increased exercise tolerance after treatment (the difference in times spent on the treadmill between baseline testing and 
after treatment) consisted solely of reduction in heart rate at 6 minutes on the treadmill $(\beta=0.543, t=4.66, \mathrm{p}<0.001)$.

\section{Discussion}

There were no significant differences in potentially confounding variables between the three groups, apart from the proportions taking antidepressants. Taking antidepressants had no significant influence on any symptomatic measure and no significant influence on strength or submaximal exercise physiology. Because antidepressants can increase the resting heart rate, ${ }^{29}$ and the greatest proportion of patients taking antidepressants were those in the group with depression, the effect of antidepressants might have been to reduce the difference we found in submaximal heart rates. Along with a type 2 error, this might explain why there was only a trend of a difference between the depressed and CFS groups in submaximal heart rates. The trend towards less height in the sedentary controls would have diminished the difference in quadriceps strength that we found.

The symptomatic data showed expected differences between the two patient groups and the healthy controls in fatigue and physical incapacity. Compared with each other, the two patient groups had similar total and mental fatigue scores, but the patients with CFS reported more physical fatigue. The CFS group was also more sensitive to noise and bodily sensations apart from pain. Compared with the other two groups the depressed patients were more depressed, anxious, mentally and emotionally incapacitated, and had worse sleep. Scores on the SF-36 provide further evidence that the pattern of impaired function is different in CFS compared with depressive illness. ${ }^{30}{ }^{31}$ As in previous studies, patients with CFS perceived greater difficulties in physical function than depressed patients, who perceived greater difficulties in mental and emotional functioning.

Exercise intolerance was demonstrated by a shorter treadmill time, lower aerobic capacity, lower maximum heart rate, and lower post-test blood lactate in both patients with CFS and depressed patients compared with healthy sedentary controls. This was not caused by respiratory dysfunction as there were no differences in resting lung function. Previous studies have also found similarly low peak heart rates during exercise tests with patients with CFS. ${ }^{12}{ }^{32}$ Riley et al also found reduced exercise capacity in patients with CFS, measured as a lower peak $\mathrm{VO}_{2}$ and shorter test duration on a Bruce treadmill test, compared with both healthy controls and patients with irritable bowel syndrome. ${ }^{6}$

To our surprise, the patients with CFS were physically weaker than both the depressed patients and sedentary subjects. No other study has found patients with CFS to be weaker than controls, ${ }^{9} 33$ although reduced quadriceps muscle strength has been reported in the related condition of fibromyalgia. ${ }^{35}$ Could this finding be artefactual due to our methods? By bypassing any CNS inhibition, the twitch interpolation technique allows a reasonable estimate of a maximal contraction. ${ }^{36}$ There was no significant difference in the proportion of subjects who needed a twitch to achieve their maximum contraction. We did not expect to find this difference so the lack of investigator blindness is unlikely to have influenced this finding. We studied a large muscle group in a large number of subjects and had to exclude the five most incapacitated subjects that we screened. We suspect that this finding of muscle weakness was related to the fact we excluded patients with CFS who also had psychiatric disorders, whereas the previous studies did not. The finding that patients with CFS were weaker (at the $6 \%$ level of probability) than depressed patients reinforces this view. Because quadriceps muscle weakness was associated with exercise capacity and was substantially reversed after graded exercise treatment, ${ }^{14}$ we think that this may be a reliable finding.

The submaximal exercise data in figs 2 and 3 show that both the patients with CFS and sedentary subjects were less physically conditioned that the depressed patients, again supporting the detrimental effect of inactivity, and reinforcing the positive influence of graded exercise therapy which helped to reverse this physical deconditioning. ${ }^{14}$ De Lorenzo et al suggested that this deconditioning of patients with CFS may be related to reduced left ventricular mass, which would explain the relative exercise induced tachycardia and extra oxygen required with exercise. ${ }^{37}$

Perceived exertion (RPE) was considerably higher in both patient groups compared with sedentary controls at submaximal stages, despite the fact that the lower oxygen consumption indicates that the exercise was less physically demanding in the depressed group. During exercise RPE is reduced after a regular training programme in both sedentary subjects $^{38}$ and patients with CFS, ${ }^{14}$ suggesting that relative inactivity or physical deconditioning increases the perception of effort during exercise. Higher perceived exertion scores in patients with CFS compared with controls have been found by others, ${ }^{6}{ }^{11-13}$ giving some credence to the hypothesis that submaximal perceived exertion is related to a central process involving a reduced threshold for afferent body sensations. ${ }^{33}$ This hypothesis is also supported by the differences in somatic amplification scores, whereby the patients with CFS showed a greater sensitivity to body sensations than the sedentary group.

The regression models of exercise tolerance (time spent on the treadmill) showed a close association with RPE, but we excluded this in our final models as, like others, we found that exercise intolerance was at least partially dependent on RPE. ${ }^{11}$ The final regression models for exercise tolerance were successfully replicated at two post-treatment times, suggesting that some reliance can be placed on the finding that quadriceps weakness and physical deconditioning were important determinants of exercise intolerance. Patients with CFS can be relatively inactive, spending more time 
resting compared with healthy subjects..$^{8}$ Increased activity consequent on graded exercise therapy improves both exercise tolerance and quadriceps strength. ${ }^{14}$ This suggests that decreased exercise tolerance and weakness in particular may be partially due to the deconditioning effects of inactivity, although an alternative explanation is possible. ${ }^{4}$ The negative association between activity and body mass index has been previously noted in subjects reporting fatigue. ${ }^{39}$ This particular association, when combined with both the known and this study's association between antidepressants and body mass index, suggests that weight reduction might be a useful treatment strategy in some patients with CFS.

This work supports a hypothesis that disability in CFS is maintained by both physical deconditioning $^{6}$ and a low threshold for certain somatic perceptions. ${ }^{40}$ Both of these factors may themselves be secondary to inactivity and causal attributions. ${ }^{10}$ The alternative interpretation is that these changes are secondary to whatever else might be causing inactivity in CFS. ${ }^{4}$ We would in any case advise caution in interpreting and generalising from these data because of the bias inherent in a case-control study, the need for replication of these data, the lack of blindness in some of the measures, and the few comparison patients with a major depressive illness.

The regression model for increased exercise tolerance supports the importance of reversing deconditioning in CFS. Although we found no significant association between feeling better after graded exercise treatment and becoming stronger or fitter, ${ }^{14}$ duration on an exercise treadmill test is a more objective measure of activity and capacity. It seemed that exposure to graded exercise therapy helped most of these patients to feel better, but improved fitness and strength were necessary for them to do more.

We are grateful for the help and advice of the following: Dr D Tunstall-Pedoe, Dr KJ Cleary, Mr J McCarthy, Ms Janice Thomas, and Miss Claire Pearson. The Linbury Trust, a Sainsbury's charitable trust, funded KYF.

1 Sharpe MC, Archard LC, Banatvala JE, et al. A report: chronic fatigue syndrome: guidelines for research. $\mathcal{F} R$ Soc Med 1991;84:118-21

2 Fukuda K, Straus S, Hickie I, et al. The chronic fatigue syndrome: a comprehensive approach to its definition and study. Ann Intern Med 1994;121:953-9.

3 Edwards RHT, Gibson H, Clague JE, et al. Muscle histopathology and physiology in chronic fatigue syndrome. In: CFS. CIBA Foundation Symposium 173 Chichester, John Wiley, 1993:102-17.

4 Lane RJ, Barret CB, Woodrow D, et al. Muscle fibre characteristics and lactate responses to exercise in chronic fatigue syndrome. F Neurol Neurosurg Psychiatry 1998;64:362-7.

5 Barnes PRJ, Taylor DJ, Kemp GJ, et al. Skeletal muscle bioenergetics in the chronic fatigue syndrome. 7 Neurol bioenergetics in the chronic fatigue
Neurosurg Psychiatry 1993;56:679-83.

6 Riley MS, O'Brien CJ, McCluskey DR, et al. Aerobic work capacity in patients with chronic fatigue syndrome. $B M \mathcal{F}$ 1990;301:953-6.

7 Wagenmakers AJM, Coakley JH, Edwards RHT. The metabolic consequences of reduced habitual activities in patients with muscle pain and disease. Ergonomics 1988;31: $1519-27$

8 Packer TL, Foster DM, Brouwer B. Fatigue and activity patterns of people with chronic fatigue syndrome. Occup Ther f Res 1997;17:186-99.
9 Vercoulen JHMM, Bazelmans E, Swanink CMA, et al. Physical activity in chronic fatigue syndrome: assessment and its role in fatigue. $\mathcal{F}$ Psychiatr Res 1997;31:661-73.

10 Vercoulen JHMM, Swanink CMA, Galama JMD, et al. The persistence of fatigue in chronic fatigue syndrome and multiple sclerosis: development of a model. F Psychosom Res 1998;45:507-17.

11 Sisto SA, LaManca J, Cordero DI, et al. Metabolic and cardiovascular effects of a progressive exercise test in patients with chronic fatigue syndrome. Am $f$ Med 1996;100:634-64.

12 Gibson H, Carroll N, Clague J, et al. Exercise performance and fatigability in patients with CFS. 7 Neurol Neurosurg and fatigability in patients
Psychiatry 1993;56:993-8.

13 Meningshoel AM, Vollestad NK, Forre O. Pain and fatigue induced by exercise in fibromyalgia patients and sedentar healthy subjects. Clin Exp Rheumatol 1995;13:477-82.

14 Fulcher KY, White PD. Randomised controlled trial of graded exercise in patients with the chronic fatigue syndrome. BMF 1997;314:1647-52.

15 Spitzer RL, Williams JB, Gibbon M, et al. Structured clinical interview for DSMIII-R: patient edition. Washington, DC: American Psychiatric Press, 1990

16 Myles WS. Sleep deprivation, physical fatigue and the perception of exercise intensity. Med Sci Sports Exerc 1985; $17: 580-4$

17 Morgan WP. Psychological components of effort sense. Med Sci Sports Exerc 1994;26:1071-7.

18 White PD, Grover SA, Kangro HO, et al. The validity and reliability of a fatigue syndrome that follows glandular fever. Psychol Med 1995;25:917-24.

19 American Psychiatric Association. Diagnostic and statistical manual for mental disorders. 3rd ed, revised version (DSMIII-R). Washington, DC: 1987.

20 Chalder T, Berelowitz G, Pawlikowska T, et al. Development of a fatigue scale. F Psychosom Res 1993;37:147-53.

21 Zigmond A, Snaith R. The hospital anxiety and depression Zigmond A, Snaith R. The hospital anxiety and

22 Buysse D, Reynolds C, Monk T, et al. The Pittsburgh sleep quality index: a new instrument for psychiatric practice and research. Psychiatry Res 1989;28:193-213.

23 Ware JE, Sherbourne CD. The MOS 36-item short form health survey (SF36). Med Care 1992;30:473-83.

24 Barsky AJ, Goodson JD, Lane RS, et al. The amplification of somatic symptoms. Psychosom Med 1988;50:510-9.

25 Vitalograph. Tables for normal values for lung function. Buckingham: Morton Press, 1980.

26 Rutherford OM, White PD. Human quadriceps strength and fatigability in patients with post viral fatigue. $\mathcal{F}$ Neurol Neurosurg Psychiatry 1991;54:961-4.

27 Borg G A. Perceived exertion rating as an indicator of somatic stress. Scand F Rehabil Med 1970;2:92-8

28 Jones DA, Newham DJ, Torgan C. Mechanical influences on long-lasting human muscle fatigue and delayed-onset muscle pain. F Physiol 1989;412:415-27.

29 Barg T, Wolferfdorf M, Ruppe A. The influence of various antidepressants on heart rate and electrodermal activity during psychophysiological examinations of depressive patients. Pharmacopsychiatry 1996;29:216-9.

30 Buchwald D, Pearlman T, Umali J, et al. Functional status in patients with chronic fatigue syndrome, other fatiguing ill-

1 Blackwood SK, MacHale SM, Power MJ, et al. Effects of exercise on cognitive and motor function in chronic fatigue syndrome and depression. I Neurol Neurosurg Psychiatry 1998;65:541-6

32 Montague TR, Marrie TJ, Klassen GA, et al. Cardiac function at rest and with exercise in the chronic fatigue syndrome. Chest 1989;95:779-84.

33 Stokes MJ, Cooper RG, Edwards RHT. Normal muscle strength and fatigability in patients with effort syndromes. BMF 1988;297:1014-17.

34 Lloyd A, Gandevia S, Hale J. Muscle performance, voluntary activation, twitch properties and perceived effort in normal subjects and patients with the chronic fatigue syndrome. Brain 1991;114:85-98.

35 Norregaard J, Bulow PM, Danneskiold-Samsoe B. Muscle strength, voluntary activation, twitch properties, and endurance in patients with fibromyalgia. $\mathcal{F}$ Neurol Neurosurg Psychiatry 1994;57:1106-11.

36 Norregaard J, Lykkegaard JJ, Bulow PM, et al. The twitch interpolation technique for the estimation of true quadriceps muscle strength. Clin Physiol 1997;17:523-32.

37 De Lorenzo F, Xiao H, Mukherjee M, et al. Chronic fatigue syndrome: physical and cardiovascular deconditioning. $Q \mathcal{F}$ Med 1998;91;475-81.

38 Ekblom B, Goldberg A. The influence of physical training and other factors in the subjective rating of perceived exerand other factors in the subjective rating of pe
tion. Acta Physiol Scand 1971;83:396-406.

39 Chen MK. The epidemiology of self-perceived fatigue among adults. Prev Med 1986;15:74-81.

40 Lawrie SM, MacHale SM, Power MJ, et al. Is the chronic fatigue syndrome best understood as a primary disturbance of the sense of effort? Psychol Med 1997;27:995-9. 